\title{
Myelin Oligodendrocyte Glycoprotein 35-55 (MOG 35-55)-induced Experimental Autoimmune Encephalomyelitis: A Model of Chronic Multiple Sclerosis
}

Sakie Miyamura, Nagisa Matsuo, Kazuki Nagayasu, Hisashi Shirakawa* and Shuji Kaneko

Department of Molecular Pharmacology, Graduate School of Pharmaceutical Sciences, Kyoto University, Sakyo-ku, Kyoto 606-8501, Japan

*For correspondence: shirakaw@pharm.kyoto-u.ac.jp

\begin{abstract}
[Abstract] Multiple sclerosis (MS) is the common demyelinating disease of human central nervous system. Among mouse models available to study MS, including the cuprizone application and lysolecithin-injection models, experimental autoimmune encephalomyelitis (EAE) model is widely used so that chronic EAE model of C57BL/6J can reflect the autoimmune pathogenesis of MS well. Here we introduce the EAE model based on C57BL/6J mice, which is generated by injection of myelin oligodendrocyte glycoprotein 35-55 (MOG 35-55) as an antigen. After immunization with complete Freund's adjuvant, clinical signs and changes in body weight are observed one or two weeks later. The EAE model will continue to be useful for development of therapeutics for MS.
\end{abstract}

Keywords: Experimental autoimmune encephalomyelitis (EAE), Multiple sclerosis (MS), Chronic, Myelin oligodendrocyte glycoprotein 35-55 (MOG 35-55), C57BL/6J, Complete Freund's adjuvant (CFA), Autoimmune, Central nervous system (CNS)

[Background] Multiple sclerosis (MS) is a chronic inflammatory disorder of the central nervous system (CNS) and is thought to have autoimmune etiology (Thompson et al., 2018). Current therapeutic treatments mainly target lymphocytes; however, this can cause serious side effects and does not provide sufficient therapeutic efficacy for those with progressive MS. Therefore, animal models of MS are important for further elucidation of pathological mechanisms and discovery of new treatments. Available pathological mouse model of MS, including the experimental autoimmune encephalomyelitis (EAE) model, the cuprizone application model, and lysolecithin-injection models, each has its own advantages (Kipp et al., 2017). EAE is used widely to study MS (Ransohoff, 2012; Baker and Amor, 2014). All EAE models enable investigation of both the immune system and CNS, which are the targets of MS therapies (Mix et al., 2010). Although there are several animal models of EAE, two are common in MS research (Burrows et al., 2019). One is the C57BL/6 mouse, which is immunized with myelin oligodendrocyte glycoprotein (MOG) (Mendel et al., 1995), and the other is the SJL mouse, which is immunized with proteolipid protein (PLP) (Tuohy et al., 1988). The former develops chronic EAE (Mendel et al., 1995; Bittner et al., 2014) and is more popular because the C57BL/6J strain has abundant genomic resources (Gold et al., 2006). The latter model develops remitting and relapsing disease, which does not happen in the MOG-EAE model; therefore, this model is used as a model for relapsing-remitting MS (Tuohy et al., 1989). 
Studies of the mechanisms underlying MS and development of suitable therapies can be conducted using EAE models; however remyelination is difficult to study in EAE model, two toxic models displaying demyelination and remyelination are widely used (Ransohoff, 2012). Cuprizone, a copper chelator, kills oligodendrocytes, resulting in demyelination and remyelination (Matsushima and Morell, 2001), whereas lysolecithin, also called lysophosphatidylcholine (LPC), is toxic to the myelin sheath (Hall, 1972). The lysolecithin-injected model has an important characteristic in that demyelination can be controlled both spatially and temporally (Keough et al., 2015). By contrast, the EAE model better reflects the autoimmune pathogenesis of MS, and represents a secondary progressive form the disease model with some modifications (Tanabe et al., 2019). Although this EAE model described in this protocol can be generated easily, disease incidence and symptoms vary according to the experimental environment. Therefore, we propose a solid method that can reliably create a C57BL/6J EAE model, which is close to human pathology and highly versatile as an animal model.

\section{Materials and Reagents}

1. $2.5 \mathrm{ml}$ Luer lock syringe (Terumo, Tokyo, Japan, catalog number: SS-02LZ)

2. Three-way stopcock (Terumo, catalog number: TS-TR2K)

3. $26 \mathrm{G}$ Needles (Terumo, catalog number: NN-2613S)

4. $18 \mathrm{G}$ Needles (Terumo, catalog number: $\mathrm{NN}-1838 \mathrm{~S}$ )

5. $1 \mathrm{ml}$ Syringe (Terumo, catalog number: SS-01T)

6. $50 \mathrm{ml}$ tube (Greiner, Kremsmünster, Austria, catalog number: 227261)

7. $2 \mathrm{ml}$ tube (INAOPTIKA, Osaka, Japan, catalog number: SC-0200)

8. Mice: C57BL/6J, female, 7-12 weeks old (Japan SLC, Shizuoka, Japan)

9. $1 \mathrm{mg} / \mathrm{ml}$ Adjuvant, Complete H37 Ra (BD Difco, Franklin Lakes, NJ, catalog number: 231131)

10. M. Tuberculosis H37 Ra (BD Difco, catalog number: 231141)

11. Pertussis toxin derived from Bordetella pertussis lyophilized powder (Sigma-Aldrich, St Louis, MO, catalog number: P7208)

12. Myelin oligodendrocyte glycoprotein 35-55 (MEVGWYRSPFSRVVHLYRNGK, purity: > 95\%, Scrum, Tokyo, Japan)

13. $5 \mathrm{mg} / \mathrm{ml}$ midazolam (Dormicum, Astellas Pharma, Tokyo, Japan)

14. $5 \mathrm{mg} / \mathrm{ml}$ butorphanol (Vetorphale, Meiji-Seika Pharma, Tokyo, Japan)

15. $1 \mathrm{mg} / \mathrm{ml}$ medetomidine (Domitor, Zenoaq, Fukushima, Japan)

16. Sodium Chloride (Nakalai Tesque, Kyoto, Japan; catalog number: 31320-05)

17. Potassium Chloride (Nakalai Tesque, catalog number: 28514-75)

18. di-Sodium Hydrogenphosphate (Nakalai Tesque, catalog number: 31801-05)

19. Potassium Dihydrogenphosphate (Nakalai Tesque, catalog number: 28721-55)

20. Saline (Otsuka Pharmaceutical, Tokyo, Japan)

21. Phosphate buffered saline (see Recipes) 


\section{Equipment}

1. Mortar and pestle (Not specified)

2. Cooking scale (Tefal, Sarcelles, France; catalog number: BC2000J2)

3. Freezer

\section{Software}

1. Graph Pad Prism 5 (Graph Pad Prism Software, San Diego, CA)

\section{Procedure}

\section{Timeline}

Day 0

Step A, Preparation of anesthesia: $\sim 15$ min

Step B-D, Preparation of MOG/CFA emulsion: $\sim 60 \mathrm{~min}$

Step E, Preparation of PTx: $\sim 10$ min

Step F, Administration of MOG/CFA emulsion and PTx: $\sim 45$ min

Day 1

Step G, EAE monitoring: 1 2 min/mouse

Day 2

Step E, Preparation of PTx: $\sim 10$ min

Steps F9 and F10, Administration of PTx: $\sim 10$ min

Step G, EAE monitoring: 1 2 min/mouse

Days 3-28

Step G, EAE monitoring: 1 2 $\mathrm{min} /$ mouse

A. Preparation of the cocktail of three different anesthetic agents

Anesthesia is important to ensure subcutaneous administration of the emulsion.

1. Prepare a cocktail of three different anesthetic agents $(30 \mu \mathrm{g} / \mathrm{ml}$ medetomidine, $400 \mu \mathrm{g} / \mathrm{ml}$ midazolam, and $500 \mu \mathrm{g} / \mathrm{ml}$ butorphanol) in saline. This solution can be stored in a refrigerator at $4{ }^{\circ} \mathrm{C}$, for at least 8 weeks after mixing. For non-immunized control mice, use the same anesthesia as EAE.

a. For a $25 \mathrm{ml}$ solution, combine $2 \mathrm{ml}$ of midazolam with $2.5 \mathrm{ml}$ of butorphanol in a $50 \mathrm{ml}$ tube.

b. Add $0.75 \mathrm{ml}$ of medetomidine. 
C. Dilute to $25 \mathrm{ml}$ with saline.

B. Preparation of Complete Freund's Adjuvant (CFA)

CFA is used to increase the immunogenicity of MOG $35-55$ as an adjuvant.

1. Calculate the required amount of $M$. Tuberculosis $\mathrm{H} 37 \mathrm{Ra}$ (MT). The required amount of MT is $500 \mu \mathrm{g}$ per mouse. There will be some loss of MT during preparation and injection, so prepare 1.5 to 2 times more than needed amount.

2. Calculate the required amounts of $1 \mathrm{mg} / \mathrm{ml}$ Adjuvant, Complete $\mathrm{H} 37 \mathrm{Ra}(1 \mathrm{mg} / \mathrm{ml}$ CFA). The required amount of $1 \mathrm{mg} / \mathrm{ml}$ CFA is $100 \mu \mathrm{l}$ per mouse. There will be some loss of CFA during preparation and injection, so prepare 1.5 to 2 times more than needed amount.

3. Place the required amount of MT into a mortar and grind with a pestle to obtain a fine powder (Figure 1A).

4. Add the required amount of $1 \mathrm{mg} / \mathrm{ml}$ CFA into the mortar (Step B3; for example, add $5 \mathrm{mg}$ MT to $1 \mathrm{ml}$ of $1 \mathrm{mg} / \mathrm{ml} \mathrm{CFA}$ ) and mix to obtain a final concentration of $6 \mathrm{mg} / \mathrm{ml}$ CFA/MT mix (Figure 1B). This solution should be prepared on the day of immunization by emulsion, and can be stored at room temperature until it is used in Step D1.

A

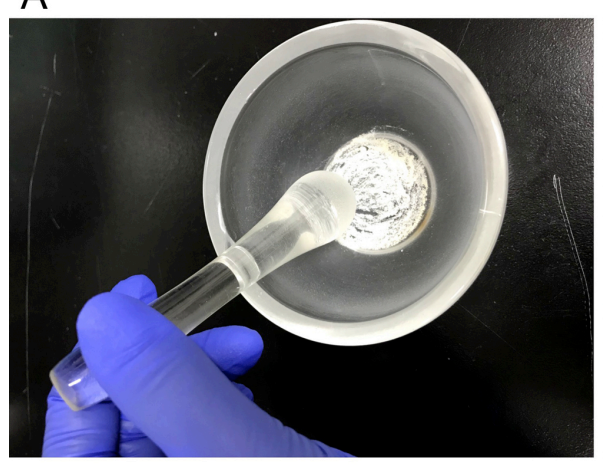

B

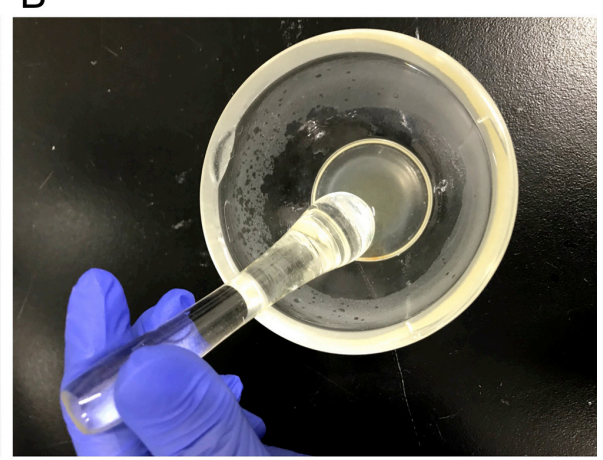

Figure 1. Preparation of Complete Freund's Adjuvant (CFA). A. Grounding MT with a mortar and a pestle. B. Mixing MT and $1 \mathrm{mg} / \mathrm{ml}$ CFA with a mortar and a pestle to obtain a final concentration of $6 \mathrm{mg} / \mathrm{ml}$ CFA/MT mix.

C. Preparation of the MOG 35-55 peptide solution

MOG 35-55 is used as an antigen to induce a demyelinating immune response.

1. Dilute lyophilized MOG $35-55$ in saline to obtain a $2 \mathrm{mg} / \mathrm{ml}$ stock solution in a $2 \mathrm{ml}$ tube. This solution ( $2 \mathrm{mg} / \mathrm{ml}$ MOG $35-55$ solution) can be stored in a freezer at $-20^{\circ} \mathrm{C}$, for at least 8 weeks after dissolution. Avoid repeated freezing and thawing.

2. Prior to immunization, dilute the MOG 35-55 stock solution with saline to yield a final concentration of $1 \mathrm{mg} / \mathrm{ml}$. This solution ( $1 \mathrm{mg} / \mathrm{ml} \mathrm{MOG} \mathrm{35-55}$ solution) should be prepared on the day of immunization by emulsion, and can be stored in a refrigerator at $4{ }^{\circ} \mathrm{C}$ until it is used in Step D4.

3. Use saline instead of MOG 35-55 solution for non-immunized control mice. 
D. Preparation of the MOG emulsion

MOG 35-55/CFA emulsion induces immune response in lymph nodes.

1. Draw $6 \mathrm{mg} / \mathrm{ml} \mathrm{CFA/MT} \mathrm{mix} \mathrm{from} \mathrm{the} \mathrm{mortar} \mathrm{(Step} \mathrm{B4)} \mathrm{into} \mathrm{a} 2.5 \mathrm{ml}$ Luer lock syringe fitted with an $18 \mathrm{G}$ needle (Figure 2A). For non-immunized control mice, use the same $6 \mathrm{mg} / \mathrm{ml}$ CFA/MT mix as EAE.

2. Remove the $18 \mathrm{G}$ needle and connect the three-way stopcock. Release the air from the stopcock (Figure 2B).

3. Turn the stopcock lever to close the valve connected to the CFA syringe (Figure $2 \mathrm{C}$ ).

4. Draw $1 \mathrm{mg} / \mathrm{ml} \mathrm{MOG} 35-55$ solution (Step C2) into another $2.5 \mathrm{ml}$ Luer lock syringe with an $18 \mathrm{G}$ needle. For non-immunized control mice, use saline instead of MOG 35-55 solution.

5. Remove the $18 \mathrm{G}$ needle and connect to the three-way stopcock. Release the air from the stopcock (Figure 2D). At this time, of either CFA or MOG solution, the liquid with the larger volume is named "solution A", and the liquid with the smaller volume is named "solution B".

6. Drain "solution A" through the three-way stopcock, so that it is the same volume as "solution B". After arranging $6 \mathrm{mg} / \mathrm{ml}$ CFA/MT mix: $1 \mathrm{mg} / \mathrm{ml} \mathrm{MOG}$ solution to $1: 1$, turn the stopcock lever to close the valve not connected to a syringe (Figure $2 \mathrm{E}$ ).

7. Pass the solution back and forth between the two syringes for about 10 min (Figure $2 F$ ). In this step, importantly, start by pushing the syringe containing the CFA at first. This will create a white emulsion. The process is complete when the syringes suddenly begin to move easily.

8. Drip just a drop of the emulsion into water to check the emulsion. If the droplet spreads on the water surface then the mixture is not ready (Figure $3 \mathrm{~A}$ ), so repeat Steps D7 and D8 until the emulsion is complete. It is ready when the droplet does not spread (Figure 3B). This emulsion should be prepared on the day of immunization, and can be stored in a refrigerator at $4{ }^{\circ} \mathrm{C}$ until use in Step F4. 
A

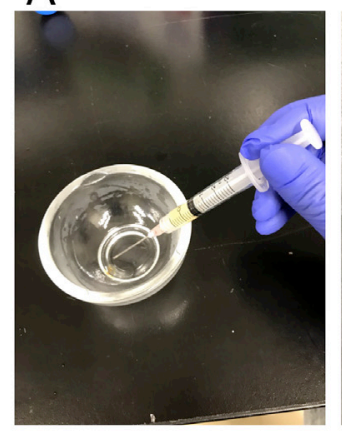

D

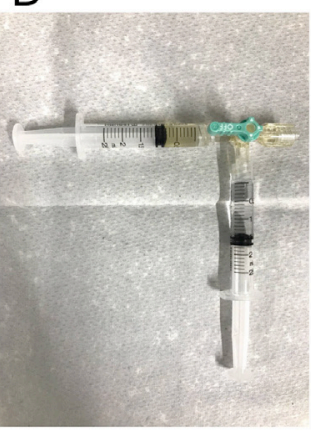

B

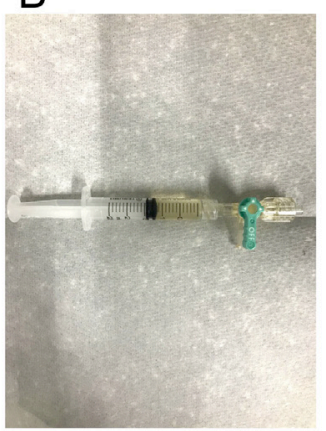

$\mathrm{E}$

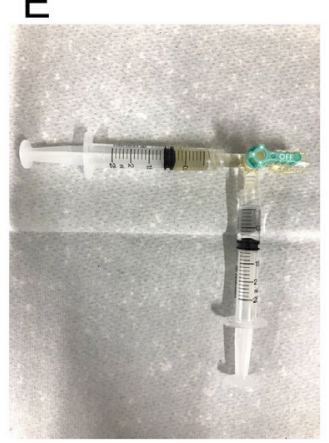

C

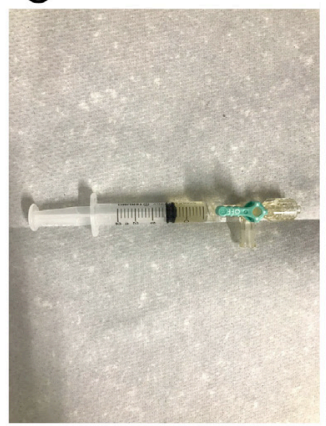

$\mathrm{F}$

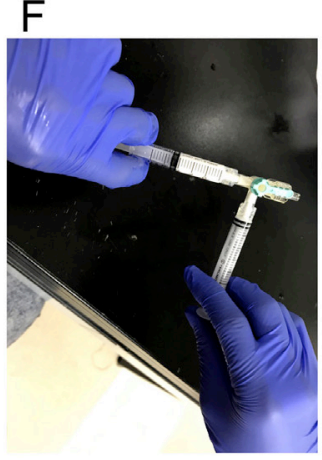

Figure 2. Preparation of the MOG emulsion. A. Drawing $6 \mathrm{mg} / \mathrm{ml} \mathrm{CFA} / \mathrm{MT}$ mix from a mortar into a $2.5 \mathrm{ml}$ leur lock syringe. B. Connecting CFA syringe to a three-way stopcock and releasing air from the stopcock. C. Closing the valve connected to the CFA syringe. D. Connecting MOG 35-55 syringe to the stopcock and releasing air. E. Arranging amount of MOG 35-55:CFA $=1: 1$ and closing the valve without syringe. F. Mixing MOG 35-55 and CFA to get white emulsion.

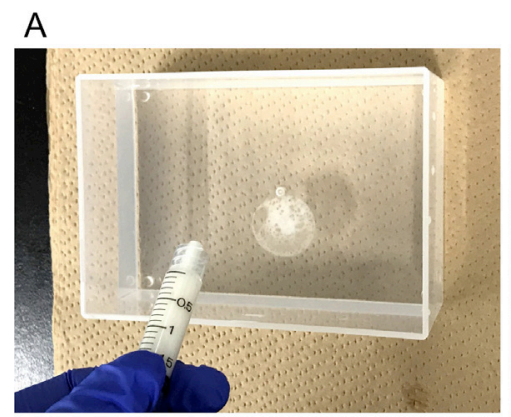

B

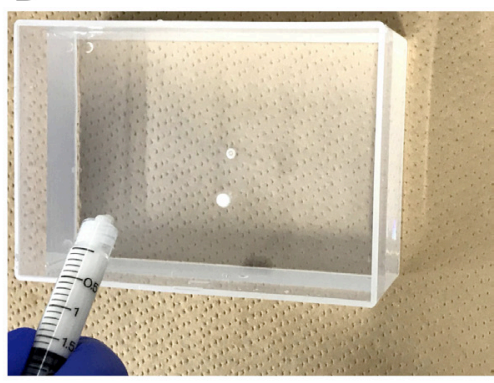

Figure 3. Checking the condition of the emulsion. A. Unfinished emulsion spread like this picture. B. Completed emulsion does not spread.

E. Preparation of Pertussis toxin (PTx)

PTx is used to disrupt the blood brain barrier and allow immune cells to invade the CNS.

1. Dilute one vial of Pertussis toxin derived from Bordetella pertussis lyophilized powder $(50 \mu \mathrm{g})$ in $1 \mathrm{ml}$ of phosphate buffered saline (PBS) to obtain a $50 \mu \mathrm{g} / \mathrm{ml} \mathrm{PTx}$ stock solution. This solution $\left(50 \mu \mathrm{g} / \mathrm{ml}\right.$ PTx stock solution) can be stored in a refrigerator at $4{ }^{\circ} \mathrm{C}$, for at least 6 months after dissolution.

2. Calculate the required amount of PTx. The required amount of PTx for each mouse is at a dose 
of $10 \mu \mathrm{g} / \mathrm{kg}$. Some Ptx will be lost during preparation and injection, so prepare 1.1 to 1.5 times more than the needed amount.

3. Dilute the PTx stock solution 1:50 in PBS to yield a final concentration of $1 \mu \mathrm{g} / \mathrm{ml}$ in a $2 \mathrm{ml}$ tube. This solution (1 $\mu \mathrm{g} / \mathrm{ml} \mathrm{Ptx}$ ) should be prepared on the day of injection (Day 0 and Day 2), and can be stored in a refrigerator at $4{ }^{\circ} \mathrm{C}$ until it is used in Steps F7 and F10. For non-immunized control mice, use the same PTx solution as EAE.

4. Prepare a $1 \mathrm{ml}$ syringe and a $26 \mathrm{G}$ needle for intraperitoneal injection.

F. Animal immunization

This immunization step is preferably performed during the light phase. Anesthesia, emulsion and PTx administration day is set to Day 0 , and PTx-only injection day is Day 2.

1. Ensure that all mice can be identified easily to enable daily evaluation, e.g., by color marking the tail base.

2. Put each mouse on the cooking scale and measure the body weight of each mouse.

3. Anesthetize with the cocktail of three different anesthetic agents. Each mouse is anesthetized by intraperitoneal (i.p.) injection $(10 \mathrm{ml} / \mathrm{kg})$. Use a $1 \mathrm{ml}$ syringe fitted with a $26 \mathrm{G}$ needle. For non-immunized control mice, use the same anesthesia as EAE. The mouse is fully anesthetized about 10 min after administration, and the anesthetic lasts about $1 \mathrm{~h}$ (Kirihara et al., 2013).

4. Remix the complete emulsion, which is prepared in Step $D$, and transfer all of the emulsion to a single syringe.

5. Remove the stopcock and connect a $26 \mathrm{G}$ needle (Figure 4A).

6. Inject $100 \mu \mathrm{l}$ of the MOG 35-55/CFA emulsion subcutaneously (s.c.) at two different sites (upper back (neck) (Figure 4B) and lower back (root of the right hind limb) (Figure 4C). For nonimmunized control mice, use the control emulsion made in Step $D$, which excludes only MOG 35-55.

7. Inject $10 \mathrm{ml} / \mathrm{kg}$ PTx solution i.p. (10 $\mu \mathrm{g} / \mathrm{kg}$ PTx). For non-immunized control mice, inject the same PTx solution as EAE.

8. Check the following points of the mice which are injected the emulsion: Whether the mouse has awakened from anesthesia, is not dead, or has an emulsion leaked from the administration site. Mice with any problems are excluded from the experiment.

9. Prepare the PTx solution as in Step E on Day 2.

10. Measure the body weight and inject a second dose of PTx on Day 2 post-immunization as in Step F7. 
A

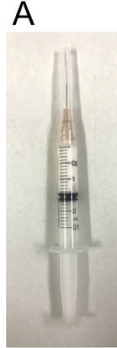

$\mathrm{B}$

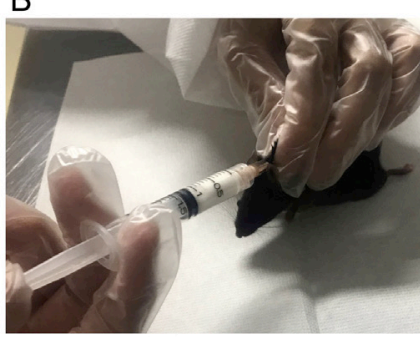

C

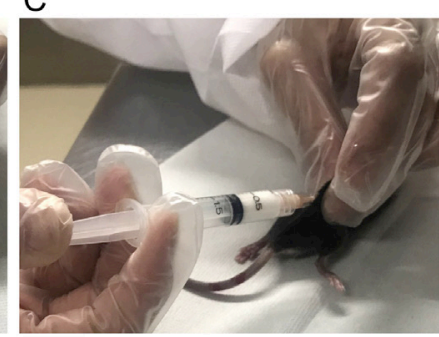

Figure 4. Animal immunization. A. Connecting a $26 \mathrm{G}$ needle to the emulsion syringe. B and C. Injecting emulsion subcutaneously to the neck $(B)$ and base of the right hindlimb $(C)$.

\section{G. EAE monitoring}

This monitoring step is preferably performed during the light phase. On Day 0 (the day of emulsion administration), the mice are evaluated after recovering from anesthesia. On Day 2 (the day of only PTx injection), the evaluation is performed immediately after administration.

1. Weigh each mouse (EAE and non-immunized control) daily.

2. On a daily basis, evaluate the clinical signs of each mouse (EAE and non-immunized control) and score as follows:
a. 0 : no clinical deficit.
b. 1: partial tail paralysis (Figure 5A, Video 1).
C. 2: full tail paralysis (Figure $5 \mathrm{~B}$, Video 2 ).
d. 3: partial hindlimb paralysis (Figure 5C, Video 3).
e. 4: full hindlimb paralysis (Figure 5D, Video 4).
f. 5: forelimb paresis (Figure $5 \mathrm{E}$, Video 5).
g. 6: dead.

\section{Details of clinical score evaluation}

First, when mouse is lifted by the center of the tail, give a score of 1 or higher if the tip of the tail hangs down and a score of 0 if not. Second, when mouse is lifted by the base of the tail, give a score of 2 or higher if the all of the tail hangs down without tension. Next, when mouse is hanged on the lid of the cage with the only forelimbs, give a score of 2 if lower body can be lifted to grab the lid not only on the forelimbs but also on the hind limbs, and a score of 3 or higher if not. Then, when mouse is put on the flat desk, give a score of 4 or higher if it drags with both hind limbs facing down, and a score of 3 if not. After that, when the lower body of a mouse is lifted, give a score of 5 if the mouse could move by the forelimbs only in one direction, either left or right, and a score of 4 if it could move in both directions. Note that non-immunized control mice do not develop such clinical symptoms at all. 
A

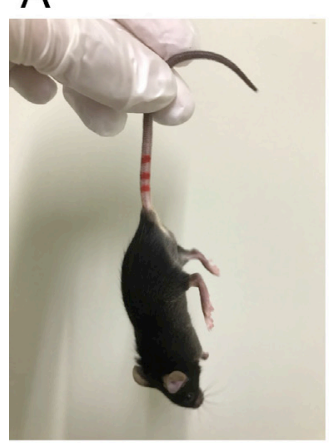

$\mathrm{D}$

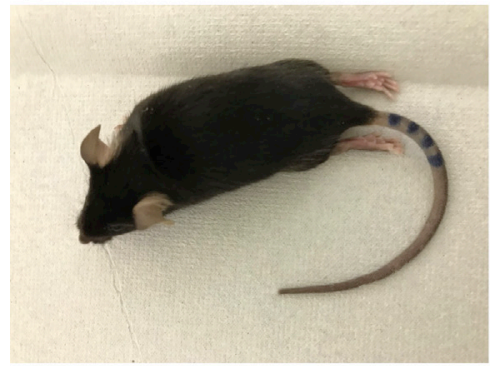

B

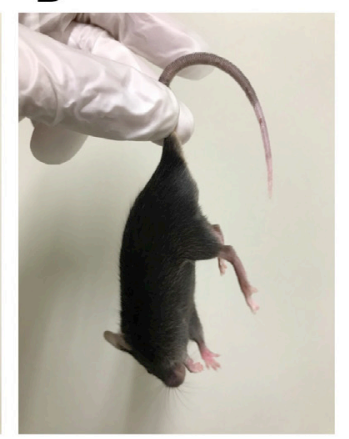

E
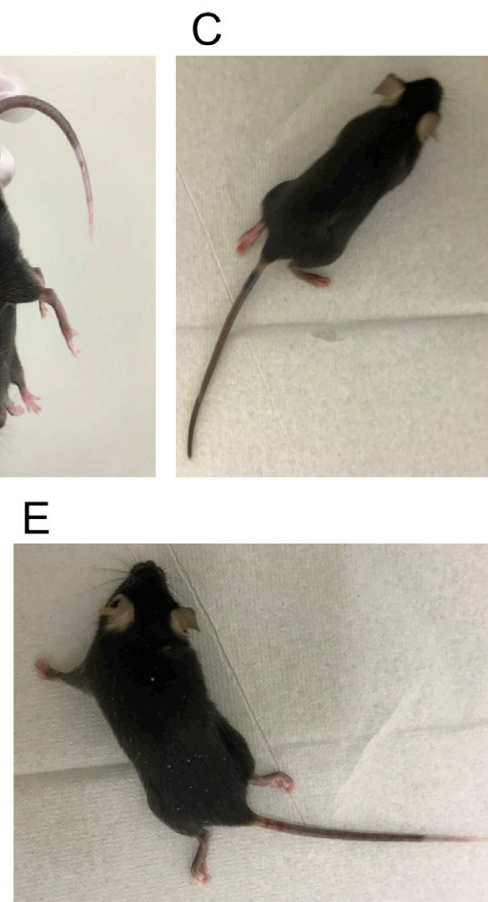

Figure 5. Clinical score evaluation of EAE mice. Representative mouse images of each clinical score. A. Clinical score 1: partial tail paralysis, B. Clinical score 2: full tail paralysis, C. Clinical score 3: partial hindlimb paralysis, D. Clinical score 4: full hindlimb paralysis, E. Clinical score 5: forelimb paresis.

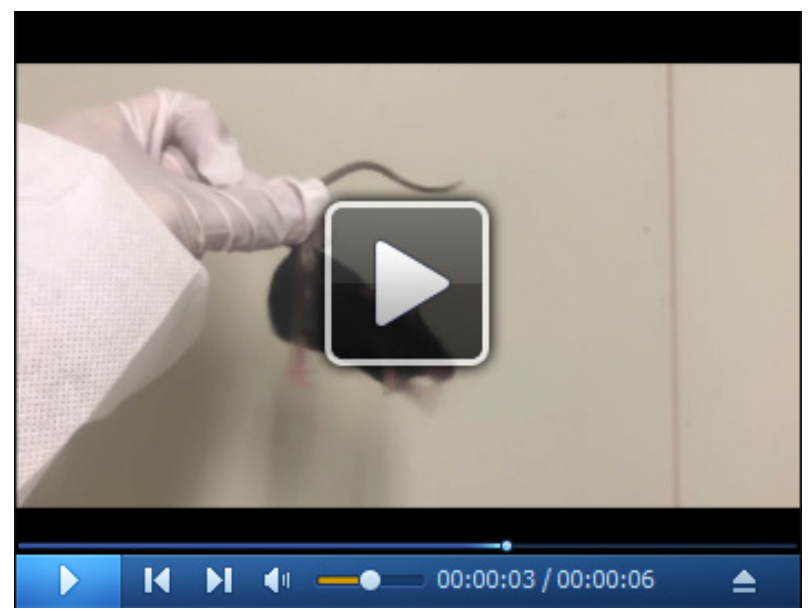

Video 1. EAE mouse with clinical score 1 


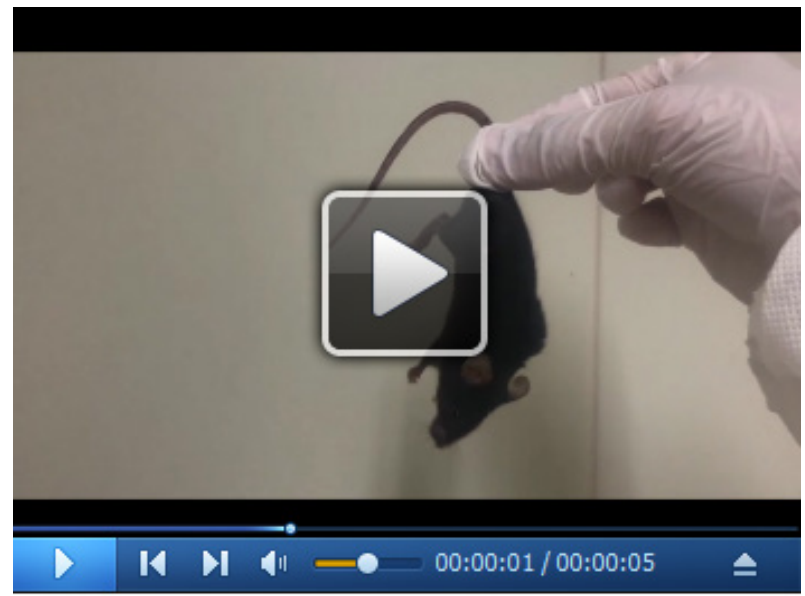

Video 2. EAE mouse with clinical score 2

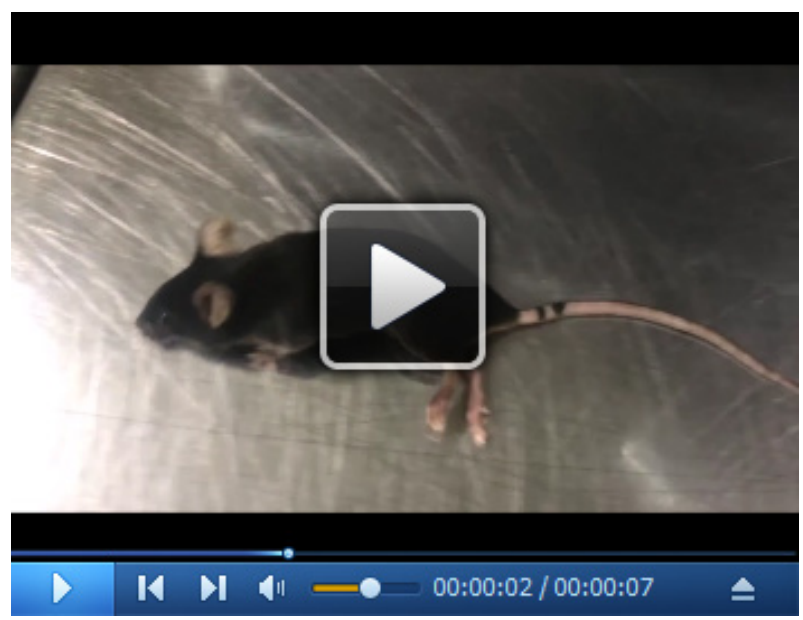

Video 3. EAE mouse with clinical score 3

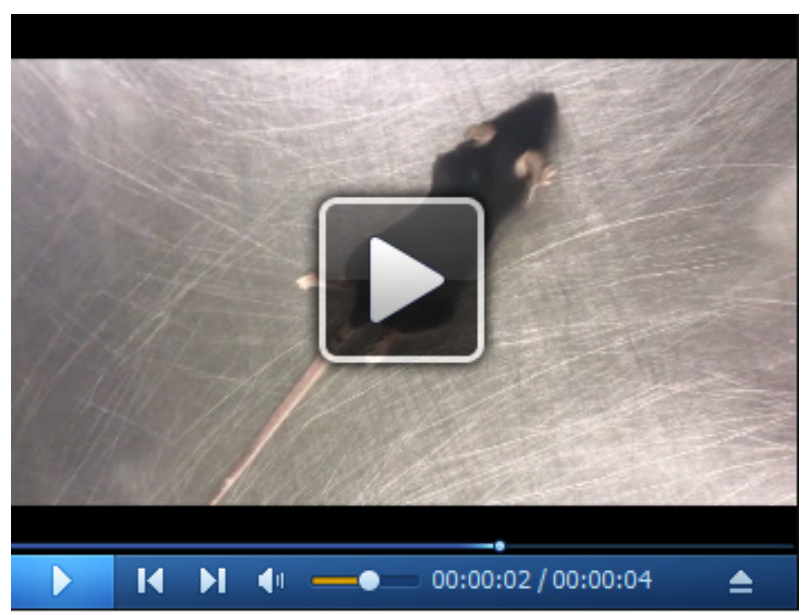

Video 4. EAE mouse with clinical score 4 


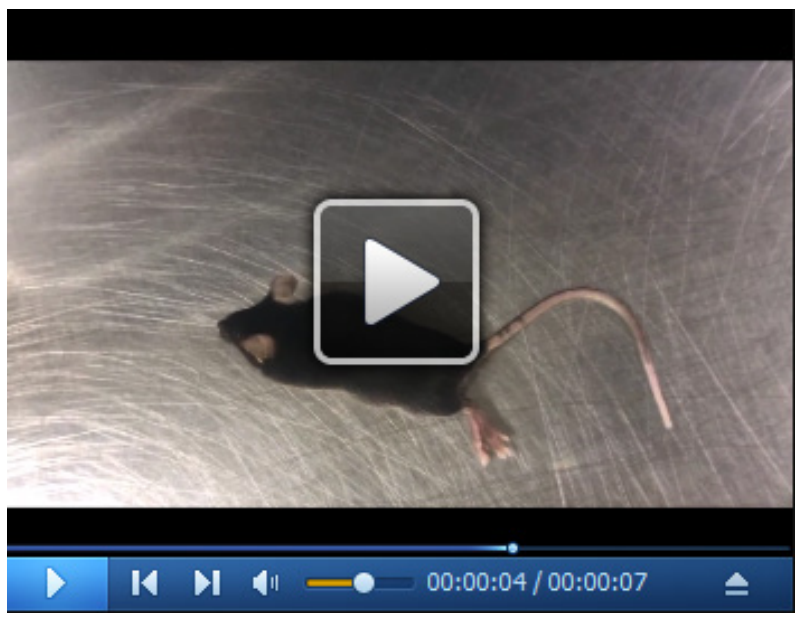

Video 5. EAE mouse with clinical score 5

These videos were made at Kyoto University in accordance with the ethical guidelines of the Kyoto University Animal Experimentation Committee and the guidelines of the Japanese Pharmacological Society, and approved by the Kyoto University Animal Experimentation Committee (Protocol Number: 19-36).

\section{Data analysis}

1. Display the daily body weight measurement data as an XY plot, where $Y$ is "Body weight" and $\mathrm{X}$ is "Days post-immunization" (Figure 6A) by using GraphPad Prism (table format: grouped).

2. Display the daily clinical score evaluation data in an XY plot, where $Y$ is the "Clinical score" and $\mathrm{X}$ is "Days post-immunization" (Figure 6B) by using GraphPad Prism (table format: grouped).

3. Analyze the data using two-way ANOVA, with Tukey's multiple-comparisons test.
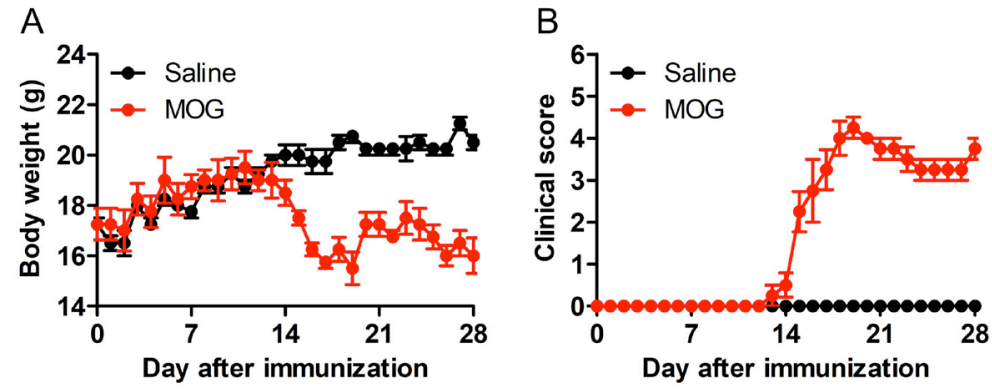

Figure 6. Changes in weight and clinical symptoms of EAE. Development of EAE in MOGimmunized C57BL/6 mice. Body weight (A) and clinical score (B) were monitored for 28 days. Note that mice began to lose body weight at the onset of clinical signs during EAE. $n=4,{ }^{* \star} P<$ $0.01,{ }^{* * *} P<0.001$. 


\section{$\underline{\text { Notes }}$}

1. Importance of grounding the MT into a fine powder (Step B3)

This step is important because the EAE incidence was higher when this operation was firmly performed compared with the careless operation. The fine powder of MT can be easily mixed with CFA, so that the EAE incidence should be increased.

2. Reasons for using a three-way stopcock to get the emulsion (Step D)

It is very important to make a strong emulsion. Therefore, we can judge the completeness of the emulsion by the slipping of syringe operation when we use a 3-way stop-cock.

3. Importance of removing the air from the stopcock (Steps D2 and D5)

If the air is not removed, the content of MOG 35-55 and CFA in the emulsion administered to one mouse could decrease, resulting in variability from experiment to experiment. Thus, the air in the three-way stopcock and the syringes should be removed.

4. Importance of starting with the CFA syringe first (Step D7)

We have tried both pushing from the CFA syringe and pushing from the MOG syringe in the step of mixing them to make an emulsion. As a result, we found that the former was easy to push when mixing by pushing the syringe, so we recommend pushing the CFA syringe first.

5. Necessity of the emulsion injection to two different sites (Step F6)

The location of each emulsion administration targets a different lymph node; the upper back (neck) is an axillary lymph node, and the lower back (root of the hind limb) is an inguinal lymph node. We think that the EAE incidence increases by causing immune responses in various lymph nodes. When the incidence is low, one of the causes could be that the administration position may be far from the lymph nodes.

6. Importance of daily body weight measurement (Step G1)

Since worsening of symptoms and weight loss correlate, it is considered that recording weight together with the clinical score leads to proper evaluation of EAE. Moreover, weight loss often occurs before the appearance of EAE clinical scores, which helps to avoid overlooking the clinical score of 1 .

7. Importance of daily clinical score evaluation (Step G2)

EAE pathological condition can be evaluated by confirming daily changing symptoms with clinical score. This symptom evaluation with drug administration or genetically modified mice leads to elucidation of the disease state and evaluation of the therapeutic effect. Thus, daily scoring is important. Clinical symptoms become apparent about 10-14 days after antigen immunization, and then reach the maximum score within a few days to a week, and often become chronic as it is. The incidence is $90-100 \%$.

8. Importance of the genetic background

Genetic background is very important because MOG 35-55 EAE model does not work in SJL mice, and PLP 139-151 EAE model does not work in C57BL/6J mice. However, NOD (nonobese diabetes) mouse is also usable for MOG EAE model. For details, see the citation of 
Stromnes and Goverman, 2006.

\section{$\underline{\text { Recipes }}$}

1. Phosphate buffered saline (PBS)

$1 \mathrm{~L}$ Ultra-pure water

$8 \mathrm{~g}$ Sodium chloride $(\mathrm{NaCl})$

$0.2 \mathrm{~g}$ Potassium chloride $(\mathrm{KCl})$

$1.15 \mathrm{~g}$ di-Sodium hydrogenphosphate $\left(\mathrm{Na}_{2} \mathrm{HPO}_{4}\right)$

$0.2 \mathrm{~g}$ Potassium dihydrogenphosphate $\left(\mathrm{KH}_{2} \mathrm{PO}_{4}\right)$

\section{Acknowledgments}

This study was supported by MEXT/JSPS KAKENHI Grant Numbers $17 K 19486$ and 19K03377 (to H.S.). The procedure was adapted from that described in a previous study (Tsutsui et al., 2018).

\section{Competing interests}

The authors declare no conflicts of interest associated with this manuscript.

\section{Ethics}

All experiments were conducted in accordance with the ethical guidelines of the Kyoto University Animal Experimentation Committee and with the guidelines of the Japanese Pharmacological Society. The approval ID of the animal experiment in this protocol is $14-42$ and the validity period is from 2014 to 2019.

\section{$\underline{\text { References }}$}

1. Baker, D. and Amor, S. (2014). Experimental autoimmune encephalomyelitis is a good model of multiple sclerosis if used wisely. Mult Scler Relat Disord 3(5): 555-564.

2. Bittner, S., Afzali, A. M., Wiendl, H. and Meuth, S. G. (2014). Myelin oligodendrocyte glycoprotein (MOG35-55) induced experimental autoimmune encephalomyelitis (EAE) in C57BL/6 mice. $J$ Vis Exp (86).

3. Burrows, D. J., McGown, A., Jain, S. A., De Felice, M., Ramesh, T. M., Sharrack, B. and Majid, A. (2019). Animal models of multiple sclerosis: From rodents to zebrafish. Mult Scler 25(3): 306324.

4. Gold, R., Linington, C. and Lassmann, H. (2006). Understanding pathogenesis and therapy of multiple sclerosis via animal models: 70 years of merits and culprits in experimental autoimmune 
encephalomyelitis research. Brain 129(Pt 8): 1953-1971.

5. Hall, S. M. (1972). The effect of injections of lysophosphatidyl choline into white matter of the adult mouse spinal cord. J Cell Sci 10(2): 535-546.

6. Keough, M. B., Jensen, S. K. and Yong, V. W. (2015). Experimental demyelination and remyelination of murine spinal cord by focal injection of lysolecithin. $J$ Vis Exp (97).

7. Kipp, M., Nyamoya, S., Hochstrasser, T. and Amor, S. (2017). Multiple sclerosis animal models: a clinical and histopathological perspective. Brain Pathol 27(2): 123-137.

8. Kirihara, Y, Takechi, M, Kurosaki, K, Kobayashi, Y and Kurosawa, T. (2013). Anesthetic effects of a mixture of medetomidine, midazolam and butorphanol in two strains of mice. Exp Anim 62(3): 173-180.

9. Matsushima, G. K. and Morell, P. (2001). The neurotoxicant, cuprizone, as a model to study demyelination and remyelination in the central nervous system. Brain Pathol 11(1): 107-116.

10. Mendel, I., Kerlero de Rosbo, N. and Ben-Nun, A. (1995). A myelin oligodendrocyte glycoprotein peptide induces typical chronic experimental autoimmune encephalomyelitis in $\mathrm{H}-2 \mathrm{~b}$ mice: fine specificity and $\mathrm{T}$ cell receptor $\mathrm{V}$ beta expression of encephalitogenic $\mathrm{T}$ cells. Eur J Immunol 25(7):1951-1959.

11. Mix, E., Meyer-Rienecker, H., Hartung, H. P. and Zettl, U. K. (2010). Animal models of multiple sclerosis--potentials and limitations. Prog Neurobiol 92(3): 386-404.

12. Ransohoff, R. M. (2012). Animal models of multiple sclerosis: the good, the bad and the bottom line. Nat Neurosci 15(8):1074-1077.

13. Stromnes, I. M. and Goverman, J. M. (2006). Active induction of experimental allergic encephalomyelitis. Nat Protoc 1(4): 1810-1819.

14. Tanabe, S., Saitoh, S., Miyajima, H., Itokazu, T. and Yamashita, T. (2019). Microglia suppress the secondary progression of autoimmune encephalomyelitis. Glia 67(9): 1694-1704.

15. Tsutsui, M., Hirase, R., Miyamura, S., Nagayasu, K., Nakagawa, T., Mori, Y., Shirakawa, H., Kaneko, S. (2018). TRPM2 exacerbates central nervous system inflammation in experimental autoimmune encephalomyelitis by increasing production of CXCL2 chemokines. $J$ Neurosci 38(39):8484-8495.

16. Thompson, A. J., Baranzini, S. E., Geurts, J., Hemmer, B. and Ciccarelli, O. (2018). Multiple sclerosis. Lancet 391(10130): 1622-1636.

17. Tuohy, V. K., Sobel, R. A. and Lees, M. B. (1988). Myelin proteolipid protein-induced experimental allergic encephalomyelitis. Variations of disease expression in different strains of mice. J Immunol 140(6): 1868-1873.

18. Tuohy, V. K., Lu, Z., Sobel, R. A., Laursen, R. A. and Lees, M. B. (1989). Identification of an encephalitogenic determinant of myelin proteolipid protein for SJL mice. J Immunol 142(5): 1523-1527. 